\section{Behaviour of myosin projections during the staircase phenomenon of heart muscle}

PEAK tension of an excised heart muscle increases with the frequency of contraction'. This 'staircase' phenomenon has been attributed to changes in the distribution of $\mathrm{Ca}$ ions; it has been proposed that the sarcoplasmic $\mathrm{Ca}^{2+}$ concentration increases with the frequency of contraction ${ }^{2-4}$. The increase in the sarcoplasmic $\mathrm{Ca}^{2+}$ is supposed to raise the peak tension by changing the behaviour of contractile proteins; however, no such molecular change has yet been clearly demonstrated. In the present study, we have investigated the behaviour of myosin projections during the staircase phenomenon by the X-ray diffraction method, in an attempt to clarify the underlying molecular events. The results have indicated that an increase in the contraction frequency causes an increase in the number of myosin projections transferred to the vicinity of the thin filaments during contraction.

A papillary muscle was isolated, together with the ventricular septum from the right ventricle of the canine heart. The muscle was cross circulated with arterial blood of a donor dog according to the method described by Endoh and Hashimoto ${ }^{5}$ : the anterior septal artery was cannulated and connected to the carotid artery of the donor dog through a perfusion pump (Harvard model 1210). The perfusion pressure was maintained at $100 \mathrm{mmHg}$. The venous outflow from the preparation was returned to the jugular vein of the donor dog.

The ventricular septum was set in a Perspex chamber surrounded by a warm bath $\left(38^{\circ} \mathrm{C}\right)$, and the papillary muscle was pulled out of the chamber through a closefitting hole. The base of the muscle was stitched to the edge of the holes. The tip of the muscle was tied to an isometric tension transducer (Shinkoh, UL). A branch of the arterial tubing was used to drip arterial blood on the surface of the papillary muscle in order to prevent the surface from cooling down and drying out. The muscle was stimulated with square pulses of $5 \mathrm{~ms}$ duration through a punctate platinum electrode ${ }^{6}$. At the beginning of each $\mathrm{X}$-ray exposure, the muscle length was adjusted to $L_{\max }$, where tension production was maximal.

A low-angle X-ray camera of the mirror-monochromator type $^{7}$ was used. A powerful source of $X$ rays was supplied by a rotating-anode generator (Rigaku, FR) operated at $50 \mathrm{kV}$ with a tube current of $70 \mathrm{~mA}$ (focal size $1 \times 0.1 \mathrm{~mm}$ ); such a high power density was made possible by using an anode of large diameter $(30 \mathrm{~cm})$ rotating at high speed ( 9,000 r.p.m.). The papillary muscie was placed vertically, and the equatorial diffraction pattern was recorded on $\mathrm{X}$-ray film (Sakura $\mathrm{N}$ ) with a specimen-to-film distance of $45 \mathrm{~cm}$. An electromagnetic shutter was placed between the monochromator and the muscle. The shutter was coupled with the tension transducer, and passed $\mathrm{X}$ rays only during the systolic phase; the shutter was opened as the tension exceeded two thirds of peak tension and closed as it fell below half of the peak tension ${ }^{8}$. Approximately 10,000 contractions were needed to obtain one equatorial pattern. As the preparation was capable of contracting more than 20,000 times with little decline of the peak tension, it was possible to record two patterns from each muscle. One of the two patterns was recorded at a contraction frequency of $120 \mathrm{~min}^{-1}$ which we chose as the 'control' frequency, and the other pattern at either 60 or $180 \mathrm{~min}^{-1}$. Each pattern was densitometered, and the integrated intensities of the 1,0 and the 1,1 reflections from the hexagonal array of the myofilaments were measured to obtain the intensity ratio $\left(I_{1,0} / I_{1,1}\right)$ (for the method of measuring the intensities ${ }^{8,9}$ ). Changes in the peak tension and the intensity ratio, caused by altering the contraction frequency in each muscle, were expressed as percentages of the values given by the same muscle at the control frequency.

At the control frequency, the peak systolic tension was $287 \pm 26 \mathrm{~g} \mathrm{~cm}^{-2}$ (mean \pm s.e.m., $n=10$ ) and the intensity ratio was $1.34 \pm 0.07 \quad(n=10)$. When the frequency was increased to $180 \mathrm{~min}^{-1}$, the peak tension increased by $26 \pm 2.7 \% \quad(n=5)$ and the intensity ratio decreased by $19 \pm 4.5 \% \quad(n=5)$. When the frequency was decreased from the control to $60 \mathrm{~min}^{-1}$, the peak tension decreased by $26 \pm 5.8 \% \quad(n=5)$ and the intensity ratio increased by $30 \pm 8.0 \% \quad(n=5)$. All these changes in the peak tension and the intensity ratio were statistically significant $(P<0.05)$.

Changes in the intensity ratio have been attributed to radial transfer of myosin projections from the vicinity of the thick filaments to that of the thin filaments ${ }^{10,11}$; a decrease in the intensity ratio indicates an increase in the number of projections in the vicinity of the thin filaments. Therefore, the present results suggest that the rise of the peak tension caused by increasing the frequency of contraction is accompanied by an increase in the number of myosin projections associated with the thin filaments during the systolic phase.

We thank Drs Edward O'Brien and Pauline Bennett for helpful comments on the earlier manuscript.

\section{Matsubara* \\ N. YAGI \\ M. ENDOH}

\section{Department of Pharmacology, \\ University of Tohoku, \\ School of Medicine,}

Seiryo-machi, Sendai, Japan 980

Received 16 January; accepted 13 March 1978.

*Present address: Department of Biophysics, King's College, 26-29 Drury Lane, London WC2, UK.

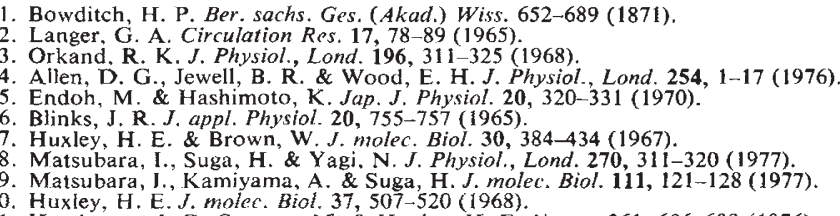

11. Haselgrove, J. C., Stewart, M. \& Huxley, H. E. Nature 261, 606-608 (1976).

\section{A temperature-sensitive yeast mitochondrial mutant with altered cytochrome $c$ oxidase subunit}

SOME of the subunits of yeast cytochrome oxidase, cytochrome $b$ and ATPase are synthesised on mitochondrial ribosomes. Inhibition of protein synthesis by antibiotics that specifically affect mitochondrial ribosomes leads to the absence of three subunits of cytochrome oxidase, at least three subunits of ATPase, and a 30,000 molecular weight polypeptide believed to be cytochrome $b$ (ref. 1). This indicated that the mitochondrion is the site of synthesis of these polypeptides, but only inferences could be drawn as to whether their mRNAs were transcribed from mitochondrial DNA (mDNA) or from nuclear DNA. We report here the isolation of a temperature-sensitive mutant of Saccharomyces cerevisiae which is mitochondrially inherited and has an altered subunit of cytochrome $c$ oxidase. The simplest interpretation of our results is that mDNA codes for this subunit.

The isolation of $\mathrm{MnCl}_{2}$-induced mitochondrial mutations specifically affecting the synthesis of cytochrome oxidase, cytochrome $b$ and ATPase has led to the possibility of directly 